\title{
LA RIVALIDAD ENTRE CHINA Y EE. UU. COMO FACTOR EN EL DESARROLLO DE LAS RELACIONES MODERNAS RUSO-ESTADOUNIDENSE
}

\author{
CHINA-US RIVALRY AS A FACTOR IN THE \\ DEVELOPMENT OF MODERN RUSSIAN-AMERICAN \\ RELATIONS
}

\section{Helen Sugelly León Ortega ${ }^{1}$ \\ ORCID: 0000-0002-1212-4137}

\section{RESUMEN}

La historia de las relaciones ruso-estadounidense, especialmente en los últimos dos siglos, muestra la permanente tensión entre los dos países junto con períodos de cooperación y entendimiento mutuo. En ese marco, este artículo indaga si las complicadas relaciones entre Estados Unidos y China en la era Trump son un factor que incide en las relaciones modernas ruso-estadounidense. Para tal propósito se realizó una investigación de carácter documental, el cual concluye que, China incide en el contexto de las relaciones ruso-estadounidenses, aunque no es un obstáculo ni un motor para el diálogo bilateral, sí ejerce una influencia significativa al presionar a Moscú para asumir una postura más activa en la formación de un nuevo orden mundial.

Palabras clave: cooperación internacional; diplomacia; política exterior; relaciones chinoestadounidenses; relaciones ruso-estadounidense; relaciones ruso-chinas.

\begin{abstract}
The history of Russian-American relations, especially in the past two centuries, shows the permanent tension between the two countries along with the limitations of cooperation and mutual understanding. In this framework, this article investigates whether the complicated relations between the United States and China in the Trump era are a factor that influences modern Russian-American relations. For this purpose, a documentary investigation was carried out, concluding that China affects the context of Russian-American relations, although it is not an obstacle or an engine for bilateral dialogue, it does exert significant influence by pressing Moscow to assume a more active position in the formation of a new world order.
\end{abstract}

Keywords: Chinese-American relations; diplomacy; foreign policy; international cooperation; Russian-American relations; RussianChinese relations.

1 Universidad Libre de Colombia, Departamento de Proyectos, Colombia. Analista internacional en HL'or Consulting. Correo: helenleon@hlorconsulting.com 


\section{Introducción}

La historia del desarrollo de las relaciones ruso-estadounidense en los siglos XX-XXI muestra que, la extensa confrontación entre los dos países, solo por un corto tiempo ha dado paso a episodios de cooperación y entendimiento mutuo. Se observó una normalidad temporal de las relaciones ruso-estadounidense durante los períodos de conflictos mundiales, y cuanto más grave fue la crisis, más exitoso resultó el desarrollo de la cooperación entre Moscú y Washington (Marcus, 2016).

Este artículo examina algunos de los factores que contribuyeron a que la comunidad internacional perciba las tensiones entre Rusia y EE. UU., con "normalidad". Junto con esto, se realiza un análisis de los cambios en el contexto internacional en la última década, que presta especial cuidado a la complicación de las relaciones entre EE. UU. y China durante la presidencia de Donald Trump, que la autora considera importante estudiar, por dos aspectos: i) la alianza estratégica integral entre Rusia y China, y, ii) el espíritu competitivo de EE. UU. frente a una Rusia aliada o en contra de una potencia mundial como China, por ejemplo en asuntos militares.

Como se verá, la influencia de factores externos puede alterar de gran manera el "equilibrio de conflicto" existente entre Rusia y EE. UU., que genera preocupación por el principio de la "asimetría distributiva", cuando la productividad de un agente mejora, en la medida en que la de otro empeora, según la teoría económica (Bernárdez, 2016). ¿Cómo cambiará este equilibrio? Al profundizar aún más las contradicciones (con la posibilidad de que Rusia se involucre en una nueva confrontación global entre EE. UU. y China) o al mejorar las relaciones del triángulo (Washington - Moscú - Beijing), que fortalece la seguridad internacional. Todo dependerá de las estrategias de política exterior elegidas por los líderes de estas potencias.

En ese mismo sentido, anota Farrés (2019) que "por tamaño y por voluntad, EE. UU. y China tienen en potencial de dar forma al sistema internacional, ya sea por coalición, o por colisión". La autora, por su parte, plantea que la eventual coalición debería incluir a Rusia para prevenir futuros conflictos en el desarrollo de las relaciones EE. UU. - China. Pero, para su implementación, Rusia debe resolver los conflictos con sus vecinos lo antes posible, establecer relaciones "amigables" con los países occidentales, y presentarse como un participante político responsable y moderado en el Sistema Internacional. 


\section{Relaciones ruso-estadounidense $\underset{i E l}{ }$ conflic- to como norma?}

En la historia de Rusia y EE. UU., se puede encontrar a estas potencias tanto como aliados como adversarios en las Relaciones Internacionales. Las crónicas en el campo de batalla, directa (como durante la Guerra Civil en Rusia) e indirectamente (como con los países del tercer mundo durante la Guerra Fría), no revelaron un ganador que dictara su voluntad a los vencidos $\mathrm{y}$, por lo tanto, en ese momento no se eliminaron los motivos para un mayor antagonismo.

Después de la división ideológica, que pasó al plano del conflicto político a fines del siglo XX, EE. UU. comenzó a imaginar a una Rusia aliada, "libre y democrática", pero en Rusia se exteriorizaron los primeros signos de EE. UU. como fuente de amenaza externa (González Oma, 2018). Sin embargo, aunque esto causó tensión, no impidió que los rusos consideraran el ícono estadounidense como una fuente de tecnología avanzada, ideal para seguir en diversas áreas (García e Ibáñez, 2017). Pero inclusive con esto, Rusia y EE. UU. no se cuentan entre los países de ideas afines con valores fundamentales comunes (Nieto, 2018).

Ahora bien, el ideal acerca del tipo de relaciones que deberían considerarse normales en torno a estas dos potencias, ha experimentado cambios significativos en comparación con las décadas de 1980 y 1990. La interacción entre Rusia y EE. UU. es tensa, Marcus estima que en Rusia existe la creencia de que Occidente nunca les ha tratado como a una nación que se libró del comunismo soviético; y más bien les ve como una patria cargada de contradicciones sistémicas, en particular de quienes toman las decisiones políticas (Sospedra, 2018).

Donald Trump, llevaba año y medio en el cargo cuando se organizó con éxito la primera reunión con Vladimir Putin; a pesar de su móvil inicial, la presión política por discrepancias en temas como Ucrania, Siria, el programa nuclear iraní, la desconfianza mutua y la competencia de armamentos, dificultaron el proceso (Zayas Rosell, 2019). La necesidad de establecer vínculos con el Kremlin facilitaba la "institucionalización" del conflicto (a lo que Vladimir Putin señala como la búsqueda del "restablecimiento de unas relaciones plenas"). El desarrollo de los sucesos ha mostrado que el formato de rivalidad estratégica es consistente con ideas sobre los intereses nacionales y el papel geopolítico de EE. UU. y Rusia en el moderno sistema de Relaciones Internacionales (Sánchez, 2018). 
Así, el mismo Vladimir Putin que cataloga las relaciones ruso-estadounidense como un factor crucial de la estabilidad global, apunta en tono diplomático que "si comprimes el resorte hasta su límite, éste recuperará su forma con más fuerza", logrando estremecer al mundo con su retórica y sus inquietantes advertencias. En todo caso, cierto es que las amenazas no solo se dirigen a Washington, sino también hacia algunos países europeos, con los que Rusia también guarda "relaciones estables", mientras no interfieran en sus intereses. Al otro lado del escenario, es evidente que Donald Trump (cuestionado por la intromisión rusa en las elecciones presidenciales de 2016), se abstiene de mostrar gestos amigables hacia su homólogo.

Lo paradójico aquí es que, aun cuando algunos temen una segunda guerra fría, sobresale una "buen" vínculo entre los dos gigantes. Y en realidad, a eso quiere referirse la autora al hablar de institucionalización del conflicto, a esta imagen de amores y odios que, de una forma u otra, ha existido durante la mayoría de los períodos históricos de las relaciones ruso-estadounidense, a este "restablecimiento de relaciones plenas", con ofensivas ocultas. Felipe Maillier (2018) lo exhibe como una tensión permanente, alimentada por la rivalidad de intereses divergentes y tramas de espionaje, que no se ha resuelto durante tanto tiempo, y que se reproducen incluso en circunstancias diferentes.

Ahora bien, merece la pena reseñar algo de lo expuesto por Marcelo Montes (2016) como el factor por el cual "conviene" desde la perspectiva norteamericana, mantener la tensión viva en el siglo XXI y, es el de debilitar el lugar de Rusia en términos económicos y tecnológicos. Esto puede parecer paradójico, pues por lo general, es la igualación de los potenciales de los competidores internacionales la que conduce al conflicto, por la percepción de amenaza. La pérdida del puesto habitual del país en la tabla clasificatoria internacional después del colapso de la URSS requirió una compensación política y psicológica, y la dura confrontación con EE. UU. se ha convertido para la Rusia moderna en una de las formas de resarcimiento (Laboire, 2016).

Surge entonces, un escenario en el que el conflicto no es un "mal absoluto", pues, como resultado de este, Rusia tuvo la oportunidad de fortalecer su nivel como potencia. Así, Rusia "parecía que en los años 2000 se aproximaría a los valores occidentales, ha virado de manera brusca en la última década y su política exterior se ha vuelto más asertiva, de manera especial en sus relaciones con Estados Unidos" (Nieto, 2018, p. 2). La capacidad de desafiar al país más poderoso, resistir la presión de las sanciones internacionales, encontrar una salida al aislamiento internacional: todo esto es una parte importante de la estrategia política de Rusia para superar el atraso objetivo de los países líderes, 
para mantener su lugar en el club de los jugadores internacionales más grandes, incluso con una ideología en desacuerdo con Occidente (Reyes Ruiz, 2018).

Entonces, aun cuando las pérdidas económicas generadas por las sanciones estimadas en USD 20000 millones al año (BBC, 2018) - y, la fuerte reducción en el acceso a préstamos y tecnologías occidentales resultaron muy agudas para Rusia, no deben minimizarse los beneficios obtenidos del antagonismo, pues en cierto sentido, como señala González Oma (2018), por el simple instinto de supervivencia, modernizaron sus fuerzas armadas, exploraron nuevas formas de contrarrestar la fuerza de EE. UU. en áreas en las que había sido dominante, al reforzar y exhibir sus ventajas tradicionales durante la última década.

En la línea de Ramírez (2016), cuando el liderazgo ruso a finales de la década de 2000 pasó de la crítica verbal de la política exterior de EE. UU., a la práctica destinada a contrarrestar los intereses estadounidenses (incluido el uso de nuevas tecnologías híbridas); casi de inmediato, el aparato político norteamericano y la opinión pública concluyeron que, el "enemigo de América" era de nuevo Rusia, como si la Guerra Fría nunca hubiera terminado, y la Unión Soviética no dejara de existir (San Martín, 2019). Para muchos gobiernos occidentales el poderío global de Rusia es producto de operaciones de pragmatismo en su máxima expresión (Magistrali, 2019).

Lo novedoso aquí es que, Donald Trump no ha confrontado directamente al gobierno de Vladimir Putin, en particular porque las relaciones con Rusia constituyen uno de los retos más importantes y quizá el mayor enigma de su política exterior. Aun cuando la idea de Rusia como enemigo resulta más comprensible y usual, que como socio o potencia regional embarcada en un proceso de democratización gradual (Ruiz, 2017). De hecho, señalan Actis y Busso (2017) que, en esta década EE. UU. identifica al terrorismo internacional y a Rusia como las principales amenazas de su superioridad global, por lo cual debe controlar "sus aventuras imperiales y expansionistas" con el fin de garantizar la supremacía en el orden internacional.

Entonces, la demanda del conflicto ruso-estadounidense como instrumento de política interna en EE. UU. y Rusia parece bastante obvia. Es la idea de la imagen del enemigo, y no la promesa de establecer vínculos estables, el medio más efectivo de atraer la atención de los votantes para la élite política de los dos países. Señalan García e Ibáñez (2017) que, es posible que las promesas de campaña de Trump de llevarse bien con Putin añadieran algunos votos en las elecciones de 2016, aun cuando los congresistas y senadores estadounidenses se han opuesto en voto unánime a Rusia; algo bastante evidente luego del paquete de sanciones aprobado en julio de 2017, que inclusive sorteaba el veto presidencial, 
por su abrumadora mayoría en el Congreso. De hecho, así como el enfoque anti-ruso se percibe en EE. UU. como el más prometedor desde el punto de vista electoral, en la Rusia posterior a Crimea, el antiamericanismo también se eleva casi al rango de religión secular y trae dividendos políticos tangibles a todos los participantes del poder existente (Ramírez, 2016).

El problema en la actualidad es que tanto Rusia como EE. UU., después de más de cien años y luego de muchos intentos de llevar una forma aceptable de coexistencia pacífica, se perciben el uno al otro como una amenaza, por sus marcadas diferencias y por su respectivo poderío, hostil a los intereses de la contraparte (Facon, 2018). Entonces, a pesar de no estar (en apariencia) interesados en una contienda, no pierden oportunidad para afirmar que se sienten preparados a plenitud para una ofensiva.

Por ello, puede argumentarse que, en el nivel de interacción bilateral el conflicto en las relaciones ruso-estadounidense es una especie de norma, tiene una estabilidad, cuya salida requiere esfuerzos serios, para lo cual Moscú y Washington apenas están preparados. Estos esfuerzos parecen superfluos, ya que ninguna de las partes ve el punto en un descenso rápido y dramático de la tensión. Es decir, el estado actual de las cosas, con toda su asimetría externa, ofrece muchas más posibilidades políticas que un acuerdo de aliados (Ramírez, 2018).

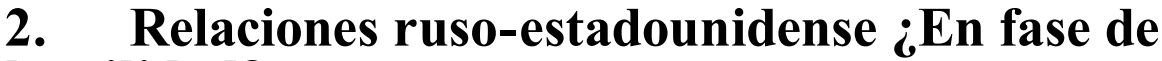 hostilidad?}

Los eventos que pueden llevar a las relaciones ruso-estadounidense a salir de una fase de hostilidad pueden ocurrir dentro de cada país o en el marco del Sistema Internacional. El escenario que funcionó durante la perestroika de Gorbachov, después de que el nuevo líder llegó al poder en la URSS y la confrontación ideológica en el marco de la Guerra Fría se disipara como humo en unos pocos años, apenas es posible en las circunstancias actuales (Rodríguez, 2019). Gorbachov intentó proponer un programa para renovar la ideología del socialismo soviético, pero subestimó la profundidad de la crisis del sistema existente, lo que condujo al colapso de la URSS y al ocaso de uno de los polos de la pugna bipolar global. Como resultado, el modelo ideológico estadounidense resultó ser de hecho el único en el mundo durante algún tiempo, experimentó un completo triunfo, reconocido incluso por la mayoría de los habitantes de la antigua URSS (Milosevich-Juaristi, 2017).

Hoy, ninguna reforma en Rusia o EE. UU., ni un nuevo líder podrían reproducir un contexto similar, máxime porque la contienda actual no está muy 
relacionada con la ideología. Rusia y EE. UU., se encuentran en un estado de hostilidad y profunda desconfianza mutua y, en las condiciones actuales, parece improbable que un "nuevo pensamiento" pueda eliminar las contradicciones políticas existentes entre ambos países (Detsch, 2018). Además, las relaciones ruso-estadounidense modernas, sin importar cómo se desarrollen, no pueden cambiar el mapa político tan rápido y de raíz como lo hizo la reconciliación de las superpotencias durante los años de la perestroika de Gorbachov. Sin demeritar la influencia de las relaciones entre Rusia y EE. UU., en la realidad actual (Collon, 2018).

Reseña Magistrali (2019) que, durante las dos primeras décadas del siglo XXI, las Relaciones Internacionales se han caracterizado por un descenso de la unipolaridad y una creciente competencia entre los países y sus asociaciones por el derecho a ser reconocidos como nuevos polos de poder. China, Rusia, India, Brasil, la Organización de Cooperación de Shanghai (OCS), la Asociación de Naciones del Sudeste Asiático (ASEAN) y muchos otros actores internacionales comenzaron a reclamar el papel de tales centros. Una de las estrategias populares para los aspirantes de influencia política internacional fue la ideología del antiamericanismo, oponiéndose a EE. UU., que, en ese momento, sin mucho éxito, intentó hacer frente a la carga del liderazgo mundial exclusivo. Pero cuanto más débil se volvió EE. UU., más errores cometió en su rol de liderazgo en el escenario externo, más fuertes fueron las críticas e incluso la oposición directa a las políticas de Washington y a los valores estadounidenses (Nieto, 2016).

La victoria en las elecciones presidenciales de 2016 de Trump fue una de las situaciones más críticas en la que EE. UU., se encontró después de un cuarto de siglo como polo dominante en la arena global. El hecho de que los votantes estadounidenses se sintieran atraídos por un candidato que abogaba por el rechazo de muchas obligaciones internacionales adquiridas por las administraciones anteriores, atestiguó el desencanto acumulado en la estrategia del activismo de política exterior. Es evidente que, el núcleo del sistema internacional existente, EE. UU., ha entrado en un estado de inestabilidad doméstica aguda (Narodowski, y Dupuy, 2017).

Tal giro político ha abierto la puerta a varios opositores internacionales de EE. UU., incluso para China, que ha estado esperando una oportunidad para transformar su influencia económica acumulada en capital político. En teoría, cualquier sobresalto en el mundo centrado en EE. UU. le da a Beijing una ventaja para fortalecer su lugar internacional dentro de la globalización (Fernández Riquelme, 2017). En un escenario en el que muchas de las iniciativas políticas de Trump fueron abiertamente anti globales, China tiene la oportunidad de jugar en contraste y tratar de presentarse como un líder alternativo a la "Nueva 
Globalización", sin el Fondo Monetario Internacional (FMI) y sin el consenso de Washington, pero también sin humillar las condiciones políticas y la interferencia de los países en asuntos de Estados soberanos (Schreiber, 2019).

Esta estrategia política de China, está cada vez más acorde con el antiamericanismo ruso. Ni la complicada historia de las relaciones, ni los desequilibrios significativos en el desarrollo económico, ni las advertencias a menudo en $\mathrm{Ru}-$ sia sobre el potencial de la amenaza china han podido evitar la proximidad entre Moscú y Beijing. La actitud hacia la formación de relaciones aliadas de facto se manifestó de clara manera en 2018-2019, después de la "Guerra comercial" entre EE. UU. y China, iniciada por el presidente Trump (Milosevich, 2019).

Durante sus reuniones, Vladimir Putin y Xi Jinping demostraron unidad en su opinión sobre todos los asuntos internacionales clave y acordaron llevar la cooperación bilateral a un nuevo nivel, con la firma de un paquete de acuerdos económicos y con el espaldarazo a la tecnología 5G de Huawei en Rusia (Canosa y Viani, 2019). Esto fue percibido por los estadounidenses como evidencia de alianza ruso-china en contra de EE. UU. Y al parecer, es de hecho la naturaleza del desarrollo de las relaciones entre EE. UU. y China lo que se convertirá en el factor más importante en el desarrollo de las relaciones entre EE. UU. y Rusia a corto y mediano plazo. Entre tanto, hay varios aspectos a considerar (Zayas Rosell, 2019).

Primero, al decidir sobre un conflicto abierto con China, el presidente Trump en definitiva transfirió la dirección de las relaciones bilaterales hacia un problema clave de política exterior y de seguridad nacional. Tanto para Trump como para sus sucesores en la Casa Blanca, la importancia de las relaciones con China ahora superará objetivamente el peso de todas las demás interacciones internacionales (Farrés, 2019). Entre otras cosas, esto crea las condiciones para reducir las pasiones en torno a Rusia, la alternativa gradual del actual conflicto ruso-estadounidense, pasa entonces de una fase aguda a una fase lenta (del Pozo, 2016).

En segundo lugar, la perspectiva de fortalecer el entendimiento mutuo entre EE. UU. y Rusia en territorio anti-chino tampoco parece viable. Lo que sí puede considerarse es el deseo de representantes de diferentes partes del espectro político en Washington de hacer todo lo posible para evitar la formación del "eje" antiamericano Beijing - Moscú. Varios de los especialistas consultados, entre ellos Facon (2018) y Schreiber (2019), señalan que, la influencia estadounidense puede tornarse negativa si la amistad entre Moscú y Beijing continúa creciendo y quizá una buena estrategia sería jugar primero la carta rusa, para sacarlos del abrazo chino; en especial porque, desde el comienzo de su gobierno, Trump 
ha visto a China como un oponente a su objetivo, desde la campaña, de hacer "nuevamente grande a América" (Magistrali, 2019).

En tercer lugar, en el nuevo entorno, el deterioro de las relaciones con EE. UU. obligará a China a desarrollar de manera más activa el componente antiamericano de las relaciones con Rusia, que hasta hace poco había sido relegado a un segundo plano por Beijing. Esto, por un lado, puede ser bien recibido por el liderazgo ruso, pero puede resultar no beneficioso para los intereses rusos. El hecho es que una alianza de pleno derecho entre China y Rusia, no se limita a la retórica sobre la amistad y los ejercicios militares conjuntos, sino que implica que una estrategia coordinada de la política exterior, puede existir hoy solo como un pacto de socios "líderes y liderados". Naturalmente, debido a las circunstancias económicas y demográficas prevalecientes, Rusia, en este modelo, tendría el papel de un socio menor, que, por supuesto, es inaceptable para las élites y la opinión pública en Rusia. Por lo anterior, es claro que hoy resultan más inmediatos los intereses de China, que quiere atraer a Rusia como uno de los instrumentos de presión sobre EE. UU. y no al revés (de la Gándara Frieyro, 2018).

En otras palabras, los acontecimientos de los últimos años en las relaciones entre EE. UU. y China en su conjunto, crean la base para un nuevo movimiento del péndulo de las relaciones ruso-estadounidense hacia una mayor comprensión mutua. Sin embargo, este movimiento es solo hipotético, y existen muchos obstáculos para su práctica. La ideología de la política exterior que ha surgido durante la presidencia de Vladimir Putin se basa en el postulado de la hostilidad de Occidente, dirigida por EE. UU. contra los intereses nacionales de Rusia (Fornillo, 2017). Por supuesto, Rusia tiene una rica experiencia en el rápido cambio de hitos ideológicos bajo el apremio de las circunstancias cambiantes, por el pragmatismo en cabeza de Putin, pero parece que el antiamericanismo moderno ha penetrado tanto en la conciencia pública que será muy difícil desarraigarlo. En este sentido, es muy diferente del antiamericanismo soviético, que se superó "en poco tiempo" como resultado de la perestroika y el lema de Gorbachov de "nuevo pensamiento político" (Mestre-Jordá, 2018).

Por otro lado, las prácticas políticas de la China moderna, hasta hace poco, estaban condenadas en Rusia, se entendía que la rigidez e incluso la crueldad del orden existente en China no podían servir como una guía política. Según la creencia popular, mantener la estabilidad política a toda costa y expandir el control estatal sobre la sociedad en China se ha convertido en la clave para un crecimiento económico sin igual (Montes, 2016). No obstante, señala Facon (2018) que, al ahondar en las razones del "milagro económico chino", países emergentes como India o Rusia podían sacar ventaja de las enseñanzas. 
Así, a pesar de que hace solo unos años, parecía que Rusia era un país más libre que China, y que muchas de las medidas tomadas por los líderes de la República Popular China (RPC) para mantener la seguridad interna nunca entrarían en el arsenal político de los líderes rusos, durante la presidencia de Dmitry Medvedev en 2008-2012, la tesis de que "la libertad es mejor que la no libertad" por un tiempo incluso se convirtió en una doctrina política básica en Rusia. Sin embargo, en la actualidad, la brecha de valor entre Rusia y China respecto a las libertades políticas se está reduciendo muy rápido. La razón, por supuesto, fue la confrontación entre Rusia y Occidente después de la unión de Crimea, y el fortalecimiento objetivo gradual de los sentimientos conservadores dentro de la élite rusa (Trenin, 2019).

Pero un factor no menos importante en la nueva proximidad de los modelos autoritarios de poder en Rusia y China fue, por extraño que parezca, el desarrollo de la sociedad de la información, el origen de nuevas tecnologías digitales que, no solo expandieron el espacio de libertad política, sino que también crearon incentivos para fortalecer el control estatal sobre la sociedad (Bernárdez, 2016). Cabe destacar que la revolución digital fue un desafío no solo para Rusia y China, sino también para las democracias occidentales, estallaron escándalos relacionados con la invasión de los servicios de inteligencia estatal en la vida privada de la ciudadanía, con la publicación de documentos secretos y conversaciones de líderes extranjeros. Todo esto ha dañado la imagen política de EE. UU. y otros países, al reclamar el estatus de los principales defensores de las libertades políticas y los Derechos Humanos (Fernández, 2017).

Al respecto, China, mediante una política de control estatal total sobre la vida pública y el espacio de información y, gracias a muchos años de experiencia en el uso de herramientas de filtrado de información, logró crear un modelo muy efectivo de la "Internet soberana", que en el período posterior a Crimea fue objeto de un estudio minucioso (incluso de intentos de copia) por parte de las estructuras del gobierno ruso (Vera, 2018). Dado que la digitalización en sus diversas manifestaciones (las nuevas tecnologías de transferencia de datos, la ciberseguridad y el problema del control sobre el libre flujo de información), sigue siendo una de las principales tendencias de la actualidad, su influencia debe tenerse en cuenta al analizar las relaciones internacionales, incluso en el triángulo Rusia - EE. UU. - China. Parece que hoy en día el factor de la digitalización dificulta el desarrollo de las relaciones entre Moscú y Washington y, proporciona a Rusia y China algunos incentivos para ampliar la cooperación (Herráez, 2016).

El campo de la ciberseguridad continúa siendo una de las áreas en las que los logros de los especialistas rusos cumplen con los más altos estándares 
internacionales. Rusia tiene todas las oportunidades para consolidar su estatus como una de las principales potencias cibernéticas (Schreiber, 2019). Sin embargo, con todas las consecuencias positivas de esto para el avance de los intereses nacionales, es otra base fundamental para el conflicto en las relaciones ruso-estadounidense (Auza, 2012). Históricamente, las tecnologías de comunicaciones digitales, incluidas las relacionadas con el desarrollo de la Internet, han emergido con mayor éxito en los laboratorios militares y universitarios de EE. UU. Las insinuaciones de Vladimir Putin acerca de que la Internet surgió como un proyecto de la Agencia Central de Inteligencia (CIA por sus siglas en inglés), puede no ser cierta, pero en un sentido estratégico refleja el hecho de que por muchos años los estadounidenses tuvieron total control sobre el espacio digital.

$\mathrm{Si}$ a lo largo de la historia las grandes potencias libraron guerras constantes por la posesión de territorios, las realidades modernas los empujan a luchar por el espacio de la información en internet. Esto no es un buen augurio para las relaciones ruso-estadounidense, pues en Moscú y Washington ven de manera muy diferente las opciones para reglamentar los asuntos digitales (Pardo de Santaya, 2017). Al mismo tiempo, para las relaciones ruso-chinas, es poco probable que la cooperación en la esfera digital se convierta en un factor de afinidad a largo plazo.

Pero, el actual interés ruso sobre la experiencia china en la regulación de la Internet no es más que un episodio en la historia de las relaciones "digitales" entre los dos países. En el campo de la oposición conjunta a las amenazas cibernéticas entre Rusia y China, no hay mucho en común. Debido a los detalles de esta esfera, que opera en un régimen de mayor alerta, todos los jugadores nacionales del ciberespacio tienden a no unirse, sino a aislarse entre sí, por temor a interferencias externas no autorizadas. A este respecto, las autoridades rusas apenas tienen motivos para creer que los informáticos chinos representan una amenaza menor para la seguridad nacional rusa que los EE. UU. Por ello, puede decirse que la próxima etapa de la revolución digital ha llevado a un aumento general de la desconfianza mutua entre los Estados, y la cooperación en el campo de la ciberseguridad sigue dando solo los primeros pasos (Actis y Busso, 2017).

Entre los factores de contacto y repudio en las Relaciones Internacionales, las cualidades personales de los liderazgos políticos y sus simpatías y antipatías suele juegar un papel importante (Farrés, 2019). En el triángulo Rusia - EE. UU. - China, se está formando una realidad interesante. Las relaciones interpersonales entre Vladimir Putin, Donald Trump y Xi Jinping en definitiva no podían clasificarse como hostiles, pues sus contactos bilaterales tuvieron lugar en un ambiente amigable. Aun cuando esto no impidió que las relaciones de EE. UU. 
con Rusia y China alcanzaran, con Trump, su punto más bajo en varias décadas, por temas ya relacionados como la percepción de amenaza o la competencia (Claudín, 2017).

Cabe destacar que después de que Trump fuera elegido presidente de EE. UU., ahora es él quien más se interesa en las relaciones con Moscú y Beijing. Aun cuando el líder chino Xi Jinping, desde que ocupó los principales puestos del país en 2011, ha tenido una actitud mucho más moderada y expectante hacia Rusia y EE. UU., (aunque en otras áreas de la política interna y externa se ha mostrado como un reformador bastante decisivo, en especial cuando se compara con su predecesor Hu Jintao) (Bregolat, 2018).

Dado que Trump enfrenta una contienda electoral en 2020, y Putin y Xi no están experimentando ese tipo de apremio electoral, se puede esperar que EE. UU. continúe manteniendo su papel proactivo en las relaciones trilaterales. Y debido a los detalles del estilo político de Trump, sus simpatías por los líderes extranjeros, así como sus diversas iniciativas, rara vez conducen a un aumento objetivo en la comprensión y cooperación mutua, pues el presidente estadounidense a menudo actúa como un cuestionador del statu quo, al catalizar cambios que no necesariamente benefician, a mediano y largo plazo, los intereses estadounidenses (Bregolat, 2019).

La única perplejidad es que, en este sistema Trump eliminó el estado de equilibrio, que hasta hace poco se consideraba centrado en EE. UU. y basado en los principios formulados por varias generaciones de la élite de Washington. Con el liderazgo político estadounidense, Trump y su equipo, hicieron en un par de años lo que Rusia y otros de sus críticos intentaron hacer por mucho tiempo, pero con menos éxito: representar a una gran potencia como un jugador impredecible en el escenario mundial (Lalinde, 2017).

\section{Reflexiones finales}

En realidad, sería una simplificación inaceptable presentar el "factor chino" como un dominante absoluto en el desarrollo de las relaciones ruso-estadounidense, porque la interacción de Moscú y Washington, a pesar de todo, sigue siendo una de las dinámicas bilaterales más significativas de las Relaciones Internacionales en el mundo moderno. Una agenda tan rica de contactos mutuos puede presumir de muy pocos actores en la política global. Entre los puntos de esta agenda hay varios temas tradicionales que despiertan interés mutuo y contribuyen a la proximidad de los dos países: la cooperación espacial, la lucha contra el terrorismo internacional, la lucha por la no proliferación nuclear, la 
respuesta a problemas ambientales globales, entre otros. Todo en condiciones favorables, puede convertirse en una buena base para romper la confrontación (Farrés, 2019).

Sin embargo, hay más que suficientes problemas que pueden enviar las relaciones ruso-estadounidense a una nueva ronda de la espiral del conflicto. Estos son puntos críticos regionales (Ucrania, Siria, Venezuela), y una nueva etapa en la carrera armamentista estratégica, y varios tipos de conflictos de valor. ¿Por qué entonces vale la pena prestar especial cuidado al factor chino? Esto se debe a tres circunstancias. Primero, con la escala del conflicto inminente entre EE. UU. y China, se desarrolla una disputa de liderazgo en el sistema político y económico mundial. Con cualquier progreso en los eventos, Rusia, como otros participantes en interacciones internacionales, no podrá permanecer como un observador indiferente (Ríos, 2018).

En segunda instancia, la dinámica que Trump ha puesto en el desarrollo de las relaciones chino-estadounidenses, y que ahora será difícil de reducir a los próximos presidentes estadounidenses. El conflicto comercial entre EE. UU. y China ya no se está gestando, sino que consolida una nueva fase, tal vez muy tormentosa (Canosa y Viani, 2019). En tercer lugar, para Rusia el resultado de la rivalidad entre EE. UU. y China es importante no solo porque China es una de las grandes potencias y participantes en el sistema global, sino también por su cercanía a Rusia, por sus alianzas actuales. Sin embargo, es en esta singularidad y predeterminación de la amistad ruso-china por circunstancias externas, donde reside la verdadera amenaza. La alianza entre Estados puede ser sostenible y efectiva solo si tiene como base valores comunes, y no solo por el deseo general de agravar la debilitada hegemonía global (García Cantalapiedra, 2017).

La desestabilización del sistema internacional, en que Rusia tendrá que tomar decisiones inequívocas a favor de EE. UU. o China, es una de las peores pesadillas geopolíticas que puede enfrentar en las circunstancias actuales (pues el desbalance del sistema internacional hace más proclive el conflicto). No obstante, el modo combativo de desarrollo de las relaciones ruso-estadounidense, que nuevamente se ha vuelto habitual, ha resultado incluso conveniente en los últimos años. Mantener un cierto nivel de tensión en las relaciones entre las superpotencias durante la Guerra Fría cumplió en su totalidad con las realidades del mundo bipolar y estableció los parámetros para el Sistema Internacional. Ahora, contrarrestar la presión estadounidense en la etapa de unipolaridad puede también tener sentido, ya que mantiene a los líderes de Washington lejos de las decisiones más impulsivas (Vélaz Azcoiti, 2019). 
Pero en las condiciones modernas, la resistencia entre Rusia y EE. UU. ya no es autosuficiente y no ayuda a resolver problemas internacionales, sino que se está convirtiendo cada vez más en un instrumento de lucha de terceros países por un lugar en la nueva jerarquía política mundial. Al ofrecer apoyo como estrategia para contrarrestar a EE. UU., Rusia se beneficia del comportamiento chino, a la vez que reduce las perspectivas políticas y oportunidades económicas norteamericanas. Y esto es, básicamente, por los impactos que estos "apoyos" puedan tener en el tablero geopolítico, al favorecer a la principal amenaza para la primacía global norteamericana, China (Actis y Busso, 2017).

Pero entonces, ¿Qué importancia tiene el factor chino para el diálogo entre Rusia y EE. UU.? ¿Se alientan las alianzas o se crean obstáculos? ¿Y qué tan preparada está Rusia para tener en cuenta las dificultades de actuar en solitario o en compañía, y no solo buscar su propio beneficio en este triángulo? Por supuesto, hay razones más objetivas y serias para el diálogo ruso-chino, que la amistad contra EE. UU., pero aun cuando se concluye que el factor chino no es un obstáculo ni un motor para el diálogo bilateral, sí es un contexto que ejerce gran influencia. Los tres países tienen una agenda diferente, pero siempre hay probabilidad de alianzas, por lo cual en este triángulo no debería esperarse la unión de dos contra el tercero.

Porque, aunque aún es difícil decir cuál será el nuevo orden mundial, Moscú y Beijing ya tienen asegurado lugares clave en él. Entonces, directa o indirectamente, se hace un llamado a Moscú, para asumir una postura más activa en la responsabilidad global de la formación de un nuevo orden mundial. Y en tales circunstancias, no solo debe dedicarse a acciones individuales, sino a identificar áreas de cooperación, establecer mecanismos completos para una respuesta equilibrada conjunta a los desafíos externos: de estabilidad estratégica, seguridad internacional, bienestar económico y desarrollo de vínculos entre potencias $\mathrm{y}$ aliados.

\section{Referencias}

Actis, E., y Busso, A. (2017). Globalización “descarriada” y "regionalismo desconcertado" en la era Trump. Raigal, (3), 51-64.

Auza, F. J. (2012). Perspectivas y proyecciones de la administración Trump. Escuela Superior de Guerra Aérea, Ciudad Autónoma de Buenos Aires. http://190.12.101.91:80/jspui/handle/1847939/782 
BBC. (2018). Cuál es el impacto de las sanciones de Occidente a Rusia ahora que entran en vigor nuevas medidas punitivas de Estados Unidos. https://www. bbc.com/mundo/noticias-internacional-45259781

Bernárdez, L. (2016). EEUU \& la Alianza Atlántica ante el desafío de Rusia. Ejército: de tierra español, (900), 120-122.

Bregolat, E. (2018). Carta de China: ¿Hacia una guerra económica entre EEUU y China? Política exterior, 32(184), 8-12.

Bregolat, E. (2019). Carta de China: Grandes murallas. Politica exterior, 33(190), 6-12.

Canosa, N., y Viani, G. (2019). China vs. EEUU: Huawei y el núcleo de la disputa. BORDES, (13), 179-187.

Claudín, C. (2017). ¿Qué pasa con los vecinos de Rusia? Anuario internacional CIDOB, (1), 111-117.

Collon, M. (2018). ¿Hay que odiar a Rusia o reflexionar? El viejo topo, (366), 54-59.

de la Gándara Frieyro, M. (2018). La política exterior rusa en la zona post-soviética: el arco de inestabilidad Báltico-Caucásico. Revista de Pensamiento Estratégico y Seguridad CISDE, 3(1), 45-65.

del Pozo, M. (2016). La política exterior de Rusia en Oriente Medio. ¿continuidad o cambio? Revista UNISCI, (41), 139-162.

Detsch, C. (2018). Escaramuzas geoestratégicas en el «patio trasero»: China y Rusia en América Latina. Nueva Sociedad, (275), 79-91.

Facon, I. (2018). Beijing y Moscú, cómplices pero no aliados: una tregua en una relación tumultuosa. Le Monde diplomatique, (274), 11.

Farrés, O. (2019). La creciente rivalidad estratégica China-EEUU y sus ramificaciones regionales. Anuario Internacional CIDOB, (1), 203-210.

Fernández, B. L. (2017). ¿El final de la no proliferación? Política exterior, 31(177), 84-91.

Fernández Riquelme, S. (2017, 2 de febrero). Angry White Man. Donald Trump y la reacción identitaria en los EEUU. La Tribuna del País Vasco. https:// latribunadelpaisvasco.com/art/5996/-angry-white-man-donald-trump-y-lareaccion-identitaria-en-los-ee-uu-

Fornillo, B. (2017). La China de Xi Jinping y el EEUU de Trump: Tensión global y el lugar de Sudamérica. Revista de la Red de Intercátedras de Historia de América Latina Contemporánea, 4(7), 6-23.

García, C., e Ibáñez, J. (2017). Populismo y nacionalismo: la política exterior estadounidense de la Administración Trump. Balance de 100 días de gobierno. 
Anuario Ceipaz Seguridad internacional y democracia: guerras, militarización y fronteras, (9), 149-166.

García Cantalapiedra, D. (2017). La administración Trump, el ascenso de la República Popular China y el nuevo «internacionalismo independiente». Revista Española de Derecho Internacional, 69(2), 325-332.

González Oma, G. M. (2018). El nacionalismo ruso como política de estado en la era de Putin (Tesis Doctoral), Universidad Nacional de La Plata.

Herráez, P. (2016). Rusia en estado puro. Política Exterior, 30(172), 114-121.

Laboire, M. (2016). La estrategia de seguridad nacional de la federación rusa (diciembre 2015). bie3: Boletín IEEE, (1), 715-725.

Lalinde, L. M. (2017). Las relaciones entre EEUU y China: ¿Necesidad de un Cuarto Comunicado Conjunto? Rev. Jiexi Zhongguo: Análisis y pensamiento latinoamericano sobre China, (25).

Lu, T. (2019). El impacto de la manufactura china en el panorama económico internacional (Tesis de pregrado). Universidad Politécnica de Cartagena, Cartagena, España.

Maillier, F. (2018). Eterna suspicacia: Rusia y la agenda de seguridad estadounidense en tiempos de Trump. Perspectivas Revista de Ciencias Sociales, 3(5), 171-191.

Magistrali, G. L. (2019). La política de defensa como herramienta de política exterior de los EEUU en los inicios de la administración Trump (2017/2018) (tesis doctoral). Escuela Superior de Guerra Tte Grl Luis María Campos.

Marcus, J. (18 de octubre de 2016). Por qué las relaciones entre Estados Unidos y Rusia están en su peor momento desde la Guerra Fría. BBC News. https:// www.bbc.com/mundo/noticias-internacional-37693275

Mestre, J. (2018). Análisis de la eficacia de las sanciones de EE. UU. y la UE a Rusia (2014-2017). bie3: Boletín IEEE, (9), 765-782.

Milosevich-Juaristi, M. (2017). El poder de la influencia rusa: la desinformación. Real Instituto El Cano.

Milosevich, M. (2019). Oso y dragón: El vínculo estratégico entre Rusia y China en el orden internacional post unipolar. Análisis del Real Instituto Elcano (ARI), (1), 1.

Montes, M. (2016). Rusia y EEUU: 25 años de relaciones. Serie de artículos y testimonios, 128. Consejo Argentino para las Relaciones Internacionales.

Narodowski, P. (2018). La interdependencia, la estrategia china y los límites del multilateralismo. V Jornadas Nacionales de Investigación en Geografía 
Argentina-XI Jornadas de Investigación y Extensión del Centro de Investigaciones Geográficas, Tandil.

Narodowski, P., y Dupuy, H. A. (2017). Estudio de la estrategia actual de EEUU, otros estados centrales y de las corporaciones multinacionales y las nuevas condiciones generales para el desarrollo productivo de la periferia / PPID/ H028. (Proyecto de investigación). Universidad Nacional de La Plata. La Plata, Argentina.

Naughton, B. (2018). ¿Puede ir China más allá del ojo por ojo? Política exterior, 32(186), 108-121.

Nieto, M. I. (2016). Rusia y la Estrategia Global de Seguridad de la Unión Europea. Revista UNISCI, (42), 197-216.

Nieto, M. I. (2018). Las relaciones EEUU-Rusia en la era Trump. Revista UNISCI, (48), 91-128.

Pardo de Santaya, J. (2017). Rusia y EEUU, en el laberinto de Oriente Medio. bie3: Boletín IEEE, (6), 184-199.

Ramírez, M. (2016). Enfrentamiento EEUU \& China/Rusia. Los riesgos de una Tercera Guerra Mundial. IX Jornadas de Sociología. Universidad Nacional de La Plata.

Ramírez, P. (2018). La política exterior de la Federación Rusa en relación con el conflicto en Siria. ¿nacionalismo versus pragmatismo? Revista Mexicana de Análisis Político y Administración Pública, 7(1), 159-186.

Reyes Ruiz, D. (2018). Análisis del poder de la política exterior de Rusia en el campo de las relaciones económicas y comerciales (Tesis de grado), PUCE.

Ríos, X. (2018). La China de Xi Jinping. Anuario Ceipaz Ascenso del nacionalismo y el autoritarismo en el sistema internacional, 143-158.

Rodríguez, P. (2019). Rusia y Venezuela: la alianza geopolítica que preocupa a EEUU. bie3: Boletín IEEE, (14), 560-573.

Ruiz, J. T. (2017). La política exterior de EEUU hacia Rusia y sus implicaciones para Europa oriental y la relación transatlántica. En S. Sousa Ferreira (ed.), Globalización y cambios en la actual agenda de seguridad (pp. 261-290). Instituto Universitario General Gutiérrez Mellado.

Sánchez, M. J. (2018). Los conflictos árticos ruso-estadounidense. Política Internacional, 7.

San Martín, H. (2019). La Guerra Hibrida Rusa Sobre Occidente. Page Publishing Inc. 
Schreiber, C. (2019). El futuro de China y Rusia como aliados en el ciberespacio. Análisis GESI, (2), 1.

Sospedra, J. (2018). Relaciones entre EE. UU. y Rusia: ¿una nueva Guerra Fría? El momento de Trump. bie3: Boletín IEEE, (9), 314-327.

Trenin, D. (2019). Estabilidad estratégica en un mundo en cambio. Política exterior, 33(190), 108-124.

Vélaz Azcoiti, L. (2019). Comparación de la estructura económica. China versus EEUU. (Tesis de pregrado). Universidad Pública de Navarra, Navarra, España.

Vera, C. G. (2018). La reemergencia de Rusia en el mundo y América Latina. Revista Política y Estrategia, (116), 143-168.

Zayas Rosell, A. (2019). Injerencias Occidente-Rusia: Rusofília versus Rusofobia. (Tesis de pregrado). Universidad de Alicante, España. 